\title{
Routine electronic surveys: development and testing of a pilot instrument for health staff assessment of feeding at 12 months: the "Weaning at One" survey
}

\author{
L.M. Tully ${ }^{1}$, A.L. Garcia ${ }^{2}$ and C.M. Wright ${ }^{2}$ \\ ${ }^{1}$ Health Promotion Research Centre, National University of Ireland Galway, University Road, Galway and ${ }^{2}$ Human \\ Nutrition, School of Medicine, MVLS, University of Glasgow, Glasgow, UK, G31 2 ER.
}

Infant feeding recommendations aim to promote a healthy diet, but in the UK there are no sources of routine data on infant feeding patterns after age 6 weeks. The Scottish Government suggests introducing "new routinely collected data where a need has been identified" (1). NHS Greater Glasgow and Clyde (GGC) has recently introduced electronic record keeping by primary care nursing teams (Health Visitors; HVTs) and recognised the opportunity to collect whole population information on infant feeding to quality assess health promotion (HP) activities. We aimed to develop a short schedule of questions, feasible for HVTs to ask during routine visits which would inform their HP advice.

A 24 question pilot survey instrument to be completed electronically or on paper was developed using strict data entry restrictions suitable for the NHS GGC electronic record system. This service evaluation pilot was tested in three areas in GGC in Dec-January 2015/6 targeting infants in the HVT's work load aged 12 months. Of 187 responses (57\% response rate; $8 \%$ online), only $40 \%$ had breastfed $(20 \%>6 \mathrm{~m}, 7 \%>11 \mathrm{~m}) ; 16 \%$ had started solids by 4 months, $38 \%$ by 5 months. Daily consumption of sugary drinks was $26 \%$, sugary snacks $24 \%$ and commercial baby foods $30 \%$. Ten $\%$ did not eat meat, $20 \%$ did not eat fish. Sweet starchy vegetables were popular ( $42 \%$ ate daily; $92 \%$ weekly) and $86 \%$ ate leafy vegetables at least weekly. Sour fruit was least popular (19\% never ate these). Only $29 \%$ ever received vitamin supplements. Aversive feeding behaviours were uncommon. $7 \%$ of parents mentioned using 'baby-led weaning'.

Breastfeeding rates and duration remain low, but the age of first complementary feeding has increased markedly. Sugar consumption among some infants is concerning. The questionnaire was acceptable to staff and once routinely implemented will provide important information about early diet and feeding behaviour.

1 Scottish Government 2011: Improving Maternal and Infant Nutrition: A Framework for Action 